\title{
Radio Frequency Interference Suppression for Landmine Detection by Quadrupole Resonance
}

\author{
Guoqing Liu, Yi Jiang, Hong Xiong, Jian Li, and Geoffrey A. Barrall \\ Department of Electrical and Computer Engineering, University of Florida, P.O. Box 116130, Gainesville, FL 32611-6130, USA
}

Received 24 August 2004; Revised 26 June 2005; Accepted 30 June 2005

\begin{abstract}
The quadrupole resonance $(\mathrm{QR})$ technology can be used as a confirming sensor for buried plastic landmine detection by detecting the explosives within the mine. We focus herein on the detection of TNT mines via the QR sensor. Since the frequency of the QR signal is located within the AM radio frequency band, the QR signal can be corrupted by strong radio frequency interferences (RFIs). Hence to detect the very weak QR signal, RFI mitigation is essential. Reference antennas, which receive RFIs only, can be used together with the main antenna, which receives both the QR signal and the RFIs, for RFI mitigation. The RFIs are usually colored both spatially and temporally, and hence exploiting only the spatial diversity of the antenna array may not give the best performance. We exploit herein both the spatial and temporal correlations of the RFIs to improve the TNT detection performance.
\end{abstract}

Copyright ( 2006 Hindawi Publishing Corporation. All rights reserved.

\section{INTRODUCTION}

The quadrupole resonance (QR) technology has been receiving increasing attention for explosive detection in applications including landmine detection [1-4]. It can be used as a confirming sensor for buried plastic landmine detection by detecting the explosives (e.g., trinitrotoluene (TNT) and Royal Demolition eXplosive (RDX)) within the mine. In this paper, we focus on the detection of TNT via the QR sensor.

When the ${ }^{14} \mathrm{~N}$ in the TNT is excited by a sequence of pulses, it will emit a signal consisting of a sequence of echoes $[1,5]$. This signal has a unique frequency signature specific to the TNT and is referred to as the TNT QR signal. The waveform of the QR signal is known a priori to within a multiplicative constant [5].

Since the TNT QR signal frequency (around $842 \mathrm{KHz}$ [1]) is located within the amplitude modulation (AM) radio frequency band and cannot be changed by other means, the AM radio signals can appear as strong radio frequency interferences (RFIs) that can seriously degrade the QR signal detection performance in a mine field. Hence to detect the very weak $Q R$ signal, the RFI mitigation is essential.

Reference antennas, which receive RFIs only, can be used together with the main antenna, which receives both the QR signal and the RFIs, for RFI mitigation. By taking advantage of the spatial correlation of the RFIs received by the antenna array, the RFIs can be reduced significantly. However, the RFIs are usually colored both spatially and temporally, and hence exploiting only the spatial diversity of the antenna array may not give the best performance.
We exploit herein both the spatial and temporal correlations of the RFIs to improve the TNT detection performance. First, we consider exploiting the spatial correlation of the RFIs only and deploy a maximum-likelihood (ML) estimator [5] for parameter estimation; we also design a constant false alarm rate (CFAR) detector for TNT detection. Second, we adopt a multichannel autoregressive (MAR) model [6] to take into account the temporal correlation of the RFIs and devise a detector based on the model. Third, we take advantage of the temporal correlation by using a robust Capon beamformer (RCB) [7] in a two-dimensional (2D) fashion (referred to as 2D RCB) with the ML estimator [5] for improved RFI mitigation. Finally, we combine the merits of all of the three aforementioned methods for TNT detection. The effectiveness of the proposed RFI mitigation methods and the combined approach is demonstrated using the experimental data collected by Quantum Magnetics (QM), Inc.

The rest of this paper is organized as follows. In Section 2, we introduce the QR data acquisition and signal model and formulate the problem of interest. Section 3 presents our RFI mitigation approaches, which include a spatial ML scheme, a temporal MAR filter, a joint fast- and slow-time 2D RCB method, and a combination of these three approaches for improved TNT detection. Also given in Section 3 is a CFAR detector for TNT detection. Experimental examples are presented in Section 4 to illustrate the performance of the proposed approaches. Finally, Section 5 contains our conclusions. 


\section{PROBLEM FORMULATION}

Consider a QR system consisting of a main antenna and $N_{c}$ reference antennas. Each of these antennas provides a spatial data acquisition channel and the data acquisition is done simultaneously on these channels. The main antenna receives both RFIs and the QR signal and the reference antennas receive only the RFIs. The QR signal is demodulated to the direct current (DC) (i.e., zero frequency) upon digitalization in the receiver.

To detect the ${ }^{14} \mathrm{~N}$ QR response of TNT, a sequence of pulses is used in the QR system built by QM [1]. One pulse sequence consists of a positive and a negative subsequence, each of which contains a sequence of $N_{s}$ echoes called an echo train. Each echo is sampled to obtain $N_{\mathrm{f}}$ fast-time samples during the acquisition window and the corresponding sampling interval $T_{\mathrm{f}}$ is referred to as the fast-time sampling interval (in analogy to the radar terminology [8]). The corresponding samples from one echo to another form the $N_{\mathrm{s}}$ slow-time samples. The fast- and slow-time samples form an $N_{\mathrm{f}} \times N_{\mathrm{s}}$ matrix. The amplitude $\gamma\left(n_{\mathrm{s}}\right)$ of the $n_{\mathrm{s}}$ th echo decays exponentially with a time constant $T_{2}[5]$ :

$$
\gamma\left(n_{\mathrm{s}}\right)=e^{-\left(n_{\mathrm{s}}-1\right) T_{\mathrm{s}} / T_{2}}, \quad n_{\mathrm{s}}=1, \ldots, N_{\mathrm{s}},
$$

where $T_{\mathrm{s}}$ is the time interval between two adjacent echoes or the slow-time sampling interval. Equation (1) also indicates the change of the QR signal from one echo to another (or from one acquisition window to another). For the data sets, we have $N_{\mathrm{f}}=50, N_{\mathrm{s}}=54$, and the fast- and slow-time sampling intervals are $T_{\mathrm{f}}=10^{-5} \mathrm{~s}$ and $T_{\mathrm{s}}=1.15 \times 10^{-3} \mathrm{~s}$, respectively.

A pair of adjacent positive and negative pulses is referred to as a pulse loop. The pulse loop is then repeated multiple times (say $N_{\mathrm{p}}$ times), that is, the data acquisition process is repeated $N_{\mathrm{p}}$ times, with each process obtaining the same QR signal. The entire data collection process in these repeated pulse loops is called a scan. Hence, each scan obtains $N_{\mathrm{p}}$ data matrices of dimension $N_{\mathrm{f}} \times N_{\mathrm{s}}$. The data collected from the negative pulse subsequence is subtracted from that in the positive pulse subsequence. This process is referred to as deringing, which cancels out any ringing from the constantphase refocusing pulses and adds up the QR signals.

Hence, we have a $2 \mathrm{D}$ complex-valued data matrix $\mathbf{X}_{n_{\mathrm{c}}, n_{\mathrm{p}}}$ of dimension $N_{\mathrm{f}} \times N_{\mathrm{s}}$ for the $n_{\mathrm{c}}$ th antenna at the $n_{\mathrm{p}}$ th pulse loop. Therefore, the QR system acquires $N_{\mathrm{p}}$ threedimensional (3D) $\left(N_{\mathrm{c}}+1\right.$ spatial channels, $N_{\mathrm{f}}$ fast-time, and $N_{\mathrm{s}}$ slow-time samples, as shown in Figure 1) data volume at each scan location.

Since the specific QR signal frequency is down-converted to zero frequency upon digitalization in the receiver, it is convenient to come up with a data model in the frequency domain by performing the one-dimensional (1D) Fourier transform (FT) along the fast-time dimension for the data sets from each antenna and then picking up the proper frequency bins corresponding to the down-converted QR signal frequency. To do so, a windowed FT (WFT) is usually used to reduce the sidelobes (we will use a Hanning window in our experiments), and the zero-frequency bin is picked up from the main antenna while multiple frequency bins (say $N_{\mathrm{b}}$ ) around the zero frequency are collected from the reference antenna outputs. For each echo of a pulse loop, $\left(1+N_{\mathrm{c}} N_{\mathrm{b}}\right)$, spatial samples are obtained from one main and $N_{c}$ reference antennas. Hence, after picking frequency bins, we have a 2D complex-valued data matrix of dimension $\left(1+N_{\mathrm{c}} N_{\mathrm{b}}\right) \times N_{\mathrm{p}} N_{\mathrm{s}}$. Consequently, the corresponding fast-frequency-domain data model can be expressed as

$$
\mathbf{x}_{l}=\beta \mathbf{a} s_{l}+\mathbf{e}_{l}, \quad l=1, \ldots, L,
$$

where $\beta \geq 0$ is the unknown signal amplitude, $\mathbf{a}$ is a vector of length $\left(1+N_{\mathrm{c}} N_{\mathrm{b}}\right)$ with the first element being 1 and the remaining ones being zeros, due to the fact that the main antenna receives both the QR signal and RFIs while the reference antennas receive only RFIs; $s_{l}$ is the signal waveform given by $s_{l}=\gamma\left(\bmod \left[l-1, N_{\mathrm{s}}\right]+1\right)\left(\right.$ with $\bmod \left[l-1, N_{\mathrm{s}}\right]$ denoting the module of $l-1$ over $N_{\mathrm{s}}$ ); we refer to a as the steering vector and $L=N_{\mathrm{p}} N_{\mathrm{s}}$ as the number of snapshots; $\mathbf{e}_{l}$ is a vector containing the RFIs and noise. In the data model in (2), we model the sequence $\left\{\mathbf{e}_{l}\right\}_{l=1}^{L}$ as a zero-mean spatially or both temporally (slow-time) and spatially colored circularly symmetric complex Gaussian random process with an unknown and arbitrary, but fixed, spatial covariance matrix.

\section{RFI MITIGATION APPROACHES}

\subsection{Spatial ML estimator and CFAR detector}

In [5], an ML approach has been proposed for a general problem of estimating the complex-valued amplitude with known waveform and known steering vector case. Based on the data model in (2) and with an assumption that the interference-plus-noise term is a zero-mean temporally white but spatially colored Gaussian process with an unknown spatial covariance matrix $\mathbf{Q}$, the spatial ML approach [5] estimates the signal amplitude by maximizing the likelihood function of the random vectors $\left\{\mathbf{x}_{l}\right\}_{l=1}^{L}$. The normalized log-likelihood function of $\left\{\mathbf{x}_{l}\right\}_{l=1}^{L}$ is

$$
C=-\ln |\mathbf{Q}|-\operatorname{tr}\left[\mathbf{Q}^{-1} \frac{1}{L} \sum_{l=1}^{L}\left(\mathbf{x}_{l}-\beta \mathbf{a} s_{l}\right)\left(\mathbf{x}_{l}-\beta \mathbf{a} s_{l}\right)^{H}\right],
$$

where $|\cdot|$ and $\operatorname{tr}(\cdot)$ denote the determinant and the trace of a matrix, respectively, and $(\cdot)^{H}$ denotes the conjugate transpose. The ML estimate of $\beta$ can be readily obtained similarly as in [5]

$$
\hat{\beta}_{\mathrm{ML}}=\frac{\left[\operatorname{Re}\left(\mathbf{a}^{H} \mathbf{T}^{-1} \mathbf{x}_{\mathrm{s}}\right)\right]_{+}}{P_{\mathrm{s}} \mathbf{a}^{H} \mathbf{T}^{-1} \mathbf{a}},
$$

where

$$
\begin{aligned}
& P_{\mathrm{s}}=\frac{1}{L} \sum_{l=1}^{L}\left|s_{l}\right|^{2}, \\
& \mathbf{x}_{\mathrm{s}}=\frac{1}{L} \sum_{l=1}^{L} \mathbf{x}_{l} s_{l}^{*}, \\
& \mathbf{T}=\widehat{\mathbf{R}}-\frac{\mathbf{x}_{\mathrm{s}} \mathbf{x}_{\mathrm{s}}^{H}}{P_{\mathrm{s}}},
\end{aligned}
$$



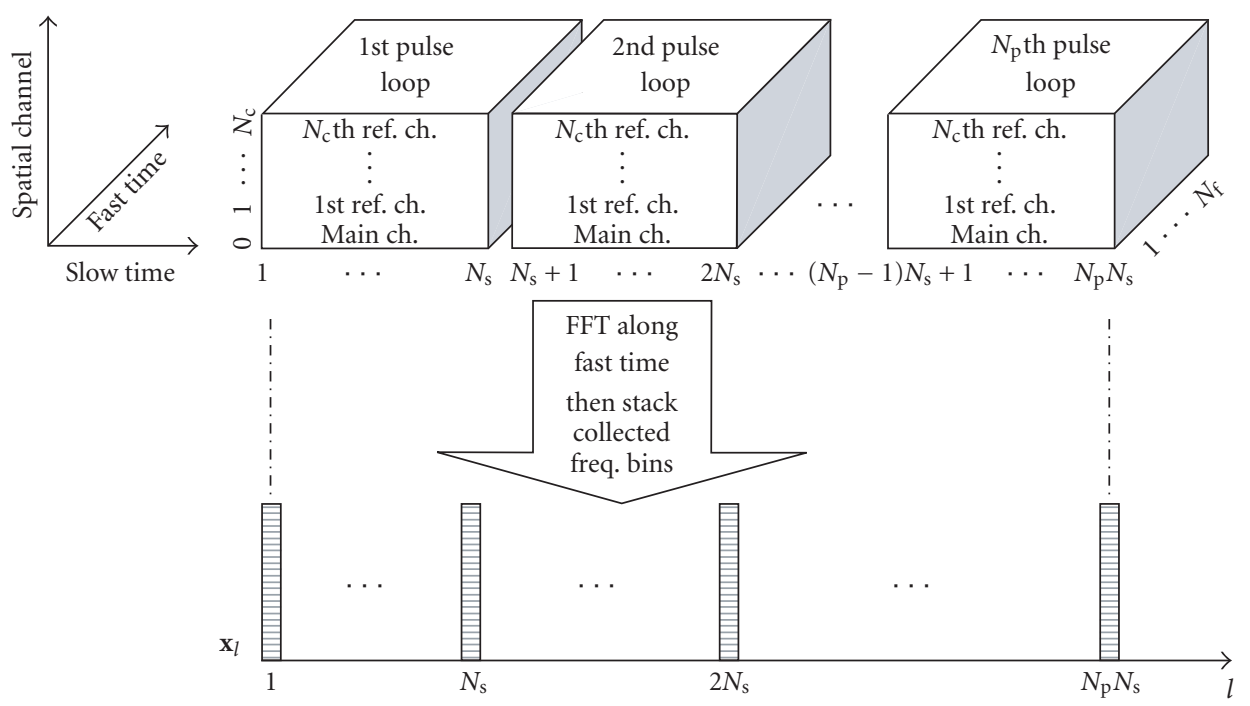

FIgUre 1: Data cube from QR data collection.

with

$$
\widehat{\mathbf{R}}=\frac{1}{L} \sum_{l=1}^{L} \mathbf{x}_{l} \mathbf{x}_{l}^{H}
$$

Here $(\cdot)^{*}$ denotes the complex conjugate, $\operatorname{Re}(\cdot)$ denotes the real part of a complex value, and $[\alpha]_{+}=\max (0, \alpha)$.

Note that the process in (4) contains three steps that have clear physical interpretations as explained below.

(1) Constructing a spatial filter:

$$
\widehat{\mathbf{w}}=\frac{\mathbf{T}^{-1} \mathbf{a}}{\mathbf{a}^{H} \mathbf{T}^{-1} \mathbf{a}} .
$$

(2) Filtering in the spatial domain:

$$
f_{l}=\widehat{\mathbf{w}}^{H} \mathbf{x}_{l}, \quad l=1, \ldots, L .
$$

(3) Filtering in the temporal domain:

$$
\hat{\beta}_{\mathrm{ML}}=\frac{1}{L P_{\mathrm{s}}}\left[\operatorname{Re}\left(\sum_{l=1}^{L} f_{l} s_{l}^{*}\right)\right]_{+} .
$$

The estimate of the signal amplitude $\hat{\beta}_{\mathrm{ML}}$ is not a sound statistic for CFAR detection because the estimated signal amplitude is highly susceptible to the environmental perturbations such as the change of the interference and noise level. For this reason, it is desired to design a detector with the CFAR behavior such that the false alarm rate is independent of the interference and noise power level. In the following, we propose an intuitive method which has the CFAR property.

After filtering the multichannel data in the spatial domain, we get a scalar sequence $\left\{f_{l}\right\}_{l=1}^{L}$ as shown in (10). The residue of the sequence $\left\{f_{l}\right\}_{l=1}^{L}$ after removing the estimated signal component $\left\{\hat{\beta}_{\mathrm{ML}} s_{l}\right\}_{l=1}^{L}$ is $\left\{f_{l}-\hat{\beta}_{\mathrm{ML}} \mathcal{s}_{l}\right\}_{l=1}^{L}$. The power of the residue is

$$
\begin{aligned}
P_{\mathrm{e}} & =\frac{1}{L} \sum_{l=1}^{L}\left|f_{l}-\hat{\beta}_{\mathrm{ML}} s_{l}\right|^{2} \\
& =\mathbf{w}^{H} \hat{\mathbf{R}} \mathbf{w}-P_{\mathrm{s}} \hat{\beta}_{\mathrm{ML}}^{2} \\
& = \begin{cases}\mathbf{w}^{H} \hat{\mathbf{R}} \mathbf{w}, & \hat{\beta}_{\mathrm{ML}}=0, \\
\frac{1}{\mathbf{a}^{H} \mathbf{T}^{-1} \mathbf{a}}+\frac{1}{P_{\mathrm{s}}} \frac{\operatorname{Im}^{2}\left(\mathbf{a}^{H} \mathbf{T}^{-1} \mathbf{x}_{\mathrm{s}}\right)}{\left(\mathbf{a}^{H} \mathbf{T}^{-1} \mathbf{a}\right)^{2}}, & \hat{\beta}_{\mathrm{ML}}>0,\end{cases}
\end{aligned}
$$

where $\operatorname{Im}(\cdot)$ denotes the imaginary part of a complex value.

We then calculate the signal-to-noise ratio (SNR) of $\left\{f_{l}\right\}_{l=1}^{L}$ as

$$
\begin{aligned}
\mathrm{SNR} & =\frac{L P_{\mathrm{s}} \hat{\beta}_{\mathrm{ML}}^{2}}{P_{\mathrm{e}}} \\
& =\frac{L\left[\operatorname{Re}\left(\mathbf{a}^{H} \mathbf{T}^{-1} \mathbf{x}_{\mathrm{s}}\right)\right]_{+}^{2}}{P_{\mathrm{s}} \mathbf{a}^{H} \mathbf{T}^{-1} \mathbf{a}+\operatorname{Im}^{2}\left(\mathbf{a}^{H} \mathbf{T}^{-1} \mathbf{x}_{\mathrm{s}}\right)} .
\end{aligned}
$$

In the appendix, we show that the test statistic defined in (13) is independent of the noise and interference scenario, and thus it is a CFAR test.

We refer to the RFI mitigation via the spatial ML approach introduced in this subsection as Method 1, which includes the following steps.

Step 1. Performing 1D WFT along fast-time dimension for the data samples from each antenna.

Step 2. Using the spatial ML estimator to obtain the ML estimate $\hat{\beta}_{\mathrm{ML}}$ of the QR signal amplitude (see (4)).

Step 3. Calculating the output SNR (see (13)).

\subsection{Temporal multichannel autoregressive Modeling}

The above spatial ML approach assumes that the interference and noise term is spatially colored but temporally white. 
However, the interference and noise (especially the RFIs) are usually spatially and temporally colored [6]. The temporal correlation can be due to the carrier of an AM radio station operating around the TNT frequency. In this case, the ML approach may perform poorly. This motivates us to consider taking into account the temporally colored interference and noise in our QR signal detection problem. In this subsection, we adopt an MAR model [6] to deal with the temporal correlation of the RFIs.

The MAR filter has the following structure [6]:

$$
\mathcal{H}\left(z^{-1}\right)=\mathbf{I}+\sum_{k=1}^{K} \mathbf{H}_{k} z^{-k},
$$

where $z^{-1}$ denotes the unit-delay operator, I is an identity matrix, and $K$ is the order of the MAR model. The MAR filter is obtained so that the output of the MAR filter in (14)

$$
\mathscr{H}\left(z^{-1}\right) \mathbf{e}_{l}=\tilde{\mathbf{e}}_{l}
$$

is temporally white.

We assume that the order $K$ of the MAR process is known (we use $K=1$ in our experiments). If $K$ is unknown, it can be estimated, for instance, by using the generalized Akaike information criterion (GAIC) [9]. The $K$ MAR coefficient matrices $\mathbf{H}=\left[\mathbf{H}_{1}, \ldots, \mathbf{H}_{K}\right]$ are estimated based on the following least-squares criterion:

$$
\begin{aligned}
\hat{\mathbf{H}}_{1}, \ldots, \hat{\mathbf{H}}_{K}=\arg \min _{\mathbf{H}_{1}, \ldots, \mathbf{H}_{K}} & \\
& \times \sum_{n_{\mathrm{p}}=1}^{N_{\mathrm{p}}} \sum_{n_{\mathrm{s}}=K+1}^{N_{\mathrm{s}}}\left\|\mathbf{e}_{n_{\mathrm{p}}, n_{\mathrm{s}}}+\sum_{k=1}^{K} \mathbf{H}_{k} \mathbf{e}_{n_{\mathrm{p}},\left(n_{\mathrm{s}}-k\right)}\right\|^{2},
\end{aligned}
$$

where $\mathbf{e}_{n_{\mathrm{p}}, n_{\mathrm{s}}}$ denotes the interference and noise term of the data model due to the $n_{\mathrm{s}}$ th slow-time sample at the $n_{\mathrm{p}}$ th pulse loop (after deringing), and $\|\cdot\|$ denotes the Euclidean norm. The solution to (16) is given by

$$
\hat{\mathbf{H}}=\mathbf{E} \Psi^{H}\left(\Psi \Psi^{H}\right)^{-1},
$$

where

$$
\begin{aligned}
\boldsymbol{\Psi} & =\left[\boldsymbol{\Psi}_{1} \cdots \boldsymbol{\Psi}_{n_{\mathrm{p}}} \cdots \boldsymbol{\Psi}_{N_{\mathrm{p}}}\right], \\
\boldsymbol{\Psi}_{n_{\mathrm{p}}} & =\left[\psi_{n_{\mathrm{p}}, K+1} \cdots \psi_{n_{\mathrm{p}}, n_{\mathrm{s}}} \cdots \psi_{n_{\mathrm{p}}, N_{\mathrm{s}}}\right], \\
\psi_{n_{\mathrm{p}}, n_{\mathrm{s}}} & =-\left[\mathbf{e}_{n_{\mathrm{p}},\left(n_{\mathrm{s}}-1\right)}^{T} \cdots \mathbf{e}_{n_{\mathrm{p}},\left(n_{\mathrm{s}}-K\right)}^{T}\right]^{T}, \\
\mathbf{E} & =\left[\mathbf{E}_{1} \cdots \mathbf{E}_{n_{\mathrm{p}}} \cdots \mathbf{E}_{N_{\mathrm{p}}}\right], \\
\mathbf{E}_{n_{\mathrm{p}}} & =\left[\mathbf{e}_{n_{\mathrm{p}}, K+1} \cdots \mathbf{e}_{n_{\mathrm{p}}, n_{\mathrm{s}}} \cdots \mathbf{e}_{n_{\mathrm{p}}, N_{\mathrm{s}}}\right] .
\end{aligned}
$$

Here $(\cdot)^{T}$ denotes the transpose.

Once the MAR filter coefficients are determined, we apply the filter to the acquired data on a per pulse-loop basis. The output of the MAR filter for the $n_{\mathrm{s}}$ th slow-time sample at the $n_{\mathrm{p}}$ th pulse loop has the form

$$
\begin{aligned}
\tilde{\mathbf{x}}_{l} & =\widehat{\mathscr{H}}\left(z^{-1}\right) \mathbf{x}_{l}=\beta \tilde{\mathbf{a}} s_{l}+\tilde{\mathbf{e}}_{l}, \\
l & =n_{\mathrm{p}} N_{\mathrm{s}}+n_{\mathrm{s}}, \quad n_{\mathrm{p}}=1, \ldots, N_{\mathrm{p}}, n_{\mathrm{s}}=K+1, \ldots, N_{\mathrm{s}},
\end{aligned}
$$

where $\widehat{\mathscr{H}}\left(z^{-1}\right)$ has the same form as $\mathscr{H}\left(z^{-1}\right)$ in (14) except that $\left\{\mathbf{H}_{k}\right\}_{k=1}^{K}$ are replaced by $\left\{\hat{\mathbf{H}}_{k}\right\}_{k=1}^{K}$,

$$
\begin{aligned}
\tilde{\mathbf{a}} & =\left(\mathbf{I}+\sum_{k=1}^{K} \hat{\mathbf{H}}_{k} e^{k T_{\mathrm{s}} / T_{2}}\right) \mathbf{a}, \\
\tilde{\mathbf{e}}_{l} & =\widehat{\mathscr{H}}\left(z^{-1}\right) \mathbf{e}_{l}, \\
l & =n_{\mathrm{p}} N_{\mathrm{s}}+n_{\mathrm{s}}, \quad n_{\mathrm{p}}=1, \ldots, N_{\mathrm{p}}, n_{\mathrm{s}}=K+1, \ldots, N_{\mathrm{s}} .
\end{aligned}
$$

Note that after the MAR filtering, the number of the slow-time samples within one pulse loop is reduced to be $N_{\mathrm{s}}-K$. Since the MAR filtering whitens the interferenceplus-noise in the temporal (slow-time) domain, the MAR filtered interference-plus-noise is still spatially colored. Also note that due to the nature of the exponentially damped QR signal waveform (see (2)), the MAR filtering does not disturb the signal waveform, and the data model in (2) is still valid for the MAR filtered data. Therefore, the spatial ML approach described in the previous section can be directly used to deal with the spatially colored MAR filtered interferenceplus-noise and to estimate the signal amplitude.

We refer to the RFI mitigation via the temporal MAR and spatial ML approach introduced in this subsection as Method 2 , which includes the following steps.

Step 1. Performing 1D WFT along the fast-time dimension for the data samples from each antenna.

Step 2. Using the temporal MAR filter to deal with the temporal correlation of the RFIs and noise.

Step 3. Applying the spatial ML estimator to the MAR filtered data and obtaining the ML estimate of the QR signal amplitude.

Step 4. Calculating the output SNR.

\subsection{Joint fast- and slow-time 2D RCB}

In this subsection, we consider using $2 \mathrm{D}$ adaptive beamforming approach and the ML estimator for the RFI mitigation. The data-adaptive standard Capon beamformer (SCB) [10] is known to have better resolution and much better interference rejection capability than the data-independent delayand-sum (DAS) beamformer [11]. SCB should perform well since the TNT QR signal is very weak. However, the performance of SCB may still degrade when the number of snapshots is small and/or nonstationary interference and noise exist. These two factors can be viewed as equivalent to steering vector errors even when the array steering vector has no error [12]. Hence instead of SCB, we use the robust Capon beamformer $(\mathrm{RCB})[7,13]$, which is a natural extension of $\mathrm{SCB}$ to the case of uncertain steering vectors, in our QR application for the RFI mitigation. Particularly, we first use 2D RCB to mitigate the RFIs jointly in the fast- and slowtime dimensions and then apply the ML approach to whiten the residual interference-and-noise in the joint temporal and spatial domain and estimate the signal amplitude.

Detailed derivations of the 1D RCB approach can be found in [7]. The extension of the $1 \mathrm{D} \mathrm{RCB}$ to the $2 \mathrm{D}$ case is given as follows (see also Figure 2). 
Input 2D matrices $\mathbf{X}_{n_{\mathrm{c}}, n_{\mathrm{p}}}$ of size $N_{\mathrm{f}} \times N_{\mathrm{s}}$

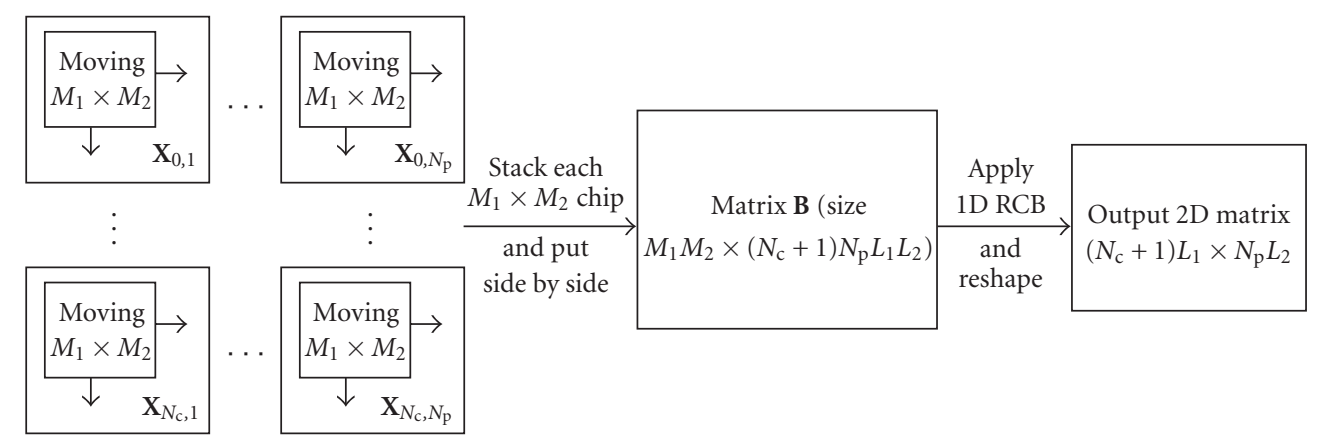

Figure 2: Flowchart of 2D RCB.

Let $M_{1}$ and $M_{2}$ be the numbers of taps in the fast- and slow-time dimensions, respectively. We choose a 2D window of size $M_{1} \times M_{2}$ and slide it downward and forward over each $2 \mathrm{D} N_{\mathrm{f}} \times N_{\mathrm{s}}$ matrix $\mathbf{X}_{n_{\mathrm{c}}, n_{\mathrm{p}}}$. At the $(m, n)$ th window location within $\mathbf{X}_{n_{\mathrm{c}}, n_{\mathrm{p}}}, m=1, \ldots, L_{1}$ with $L_{1}=N_{\mathrm{f}}-M_{1}+1$ and $n=$ $1, \ldots, L_{2}$ with $L_{2}=N_{s}-M_{2}+1$, we obtain a vector $\mathbf{b}_{n_{c}, n_{\mathrm{p}}}(m, n)$ by stacking the columns of the submatrix of $\mathbf{X}_{n_{\mathrm{c}}, n_{\mathrm{p}}}$, covered by the moving window, on top of each other. We then put all so-obtained vectors side by side and obtain a new $2 \mathrm{D}$ data matrix

$$
\mathbf{B}=\left[\mathbf{B}_{0} \cdots \mathbf{B}_{n_{\mathrm{c}}} \cdots \mathbf{B}_{\mathrm{N}_{\mathrm{c}}}\right]
$$

where

$$
\mathbf{B}_{n_{\mathrm{c}}}=\left[\mathbf{B}_{n_{\mathrm{c}}, 1} \cdots \mathbf{B}_{n_{\mathrm{c}}, n_{\mathrm{p}}} \cdots \mathbf{B}_{n_{\mathrm{c}}, N_{\mathrm{p}}}\right], \quad n_{\mathrm{c}}=0, \ldots, N_{\mathrm{c}},
$$

with

$$
\begin{gathered}
\mathbf{B}_{n_{\mathrm{c}}, n_{\mathrm{p}}}=\left[\mathbf{b}_{n_{\mathrm{c}}, n_{\mathrm{p}}}(1,1) \cdots \mathbf{b}_{n_{\mathrm{c}}, n_{\mathrm{p}}}(m, n) \cdots \mathbf{b}_{n_{\mathrm{c}}, n_{\mathrm{p}}}\left(L_{1}, L_{2}\right)\right], \\
n_{\mathrm{c}}=0, \ldots, N_{\mathrm{c}} ; n_{\mathrm{p}}=1, \ldots, N_{\mathrm{p}} .
\end{gathered}
$$

With this preparation, the 1D RCB is then applied to the new data matrix $\mathbf{B}$ whose columns are considered as snapshots. In this case, the estimated sample covariance matrix $\hat{\mathbf{R}}_{\mathrm{fs}}$ is given by

$$
\widehat{\mathbf{R}}_{\mathrm{fs}}=\frac{1}{\left(N_{\mathrm{c}}+1\right) N_{\mathrm{p}} L_{1} L_{2}} \mathbf{B B}^{H},
$$

and the desired steering vector is given by

$$
\mathbf{a}_{0}=\left[s_{1} \mathbf{1}_{M_{1}}^{T} \cdots s_{M_{2}} \mathbf{1}_{M_{1}}^{T}\right]^{T},
$$

with $\mathbf{1}_{M_{1}}$ being an all-one vector of length $M_{1}$. Note that using $\mathbf{1}_{M_{1}}$ in (25) is an approximation since the QR signal is not a constant strictly over the fast time. Figure 3 shows the TNT QR signal as a function of the fast-time sample number obtained by scanning a TNT mine in a high SNR and RFI-free experiment. RCB is robust against this approximation of the QR signal.

Given the estimated sample covariance matrix $\hat{\mathbf{R}}_{\mathrm{fs}}$ and the desired steering vector $\mathbf{a}_{0}$, RCB estimates the actual steering

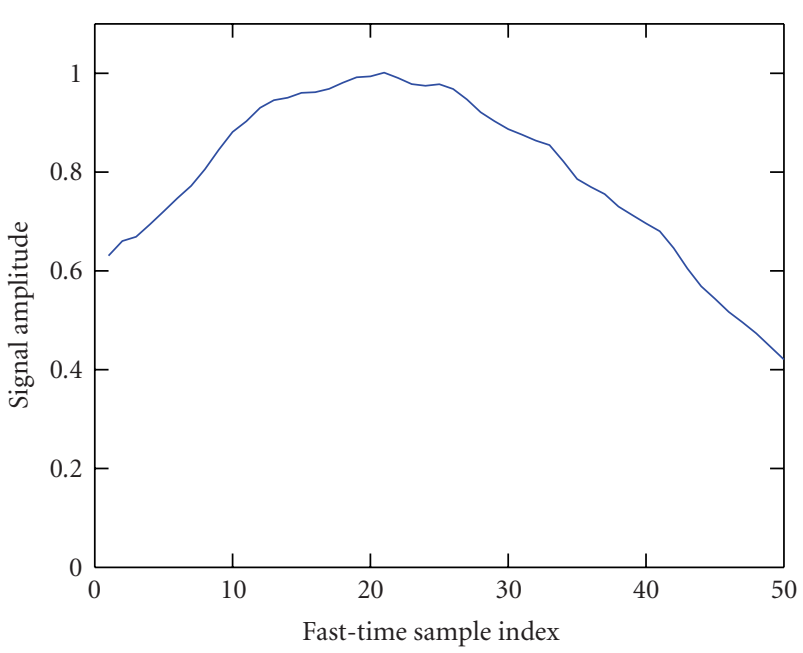

FIGURE 3: QR signal versus fast-time sample number (obtained by scanning a TNT mine in a high-SNR and RFI-free experiment).

vector $\breve{a}$ and the signal power $\sigma^{2}$ by solving the following optimization problem [7]:

$$
\begin{array}{r}
\max _{\sigma^{2}, \breve{\mathbf{a}}} \sigma^{2} \quad \text { subject to } \hat{\mathbf{R}}_{\mathrm{fs}}-\sigma^{2} \breve{\mathbf{a}} \breve{\mathbf{a}}^{H} \geq 0, \\
\left\|\breve{\mathbf{a}}-\mathbf{a}_{0}\right\| \leq \epsilon,
\end{array}
$$

where $\epsilon$ is a user parameter which is used to describe the steering vector uncertainty. Note that the first line of (26) can be interpreted as a covariance fitting problem: given $\hat{\mathbf{R}}_{\mathrm{fs}}$ and ă, we wish to determine the largest possible signal of interest $\sigma^{2} \breve{a} \breve{a}^{H}$ that can be a part of $\widehat{\mathbf{R}}_{\mathrm{fs}}$ under the natural constraint that the residual covariance matrix is positive semidefinite.

It is shown in [7] that the above optimization problem is equivalent to the following quadratic optimization under a spherical constraint:

$$
\min _{\breve{\mathbf{a}}} \breve{\mathbf{a}}^{H} \hat{\mathbf{R}}_{\mathrm{fs}}^{-1} \breve{\mathbf{a}} \quad \text { subject to }\left\|\breve{\mathbf{a}}-\mathbf{a}_{0}\right\|^{2}=\epsilon .
$$


This optimization problem can be solved by using the Lagrange multiplier methodology, which is based on the function

$$
f=\breve{\mathbf{a}}^{H} \hat{\mathbf{R}}_{\mathrm{fs}}^{-1} \breve{\mathbf{a}}+\lambda\left(\left\|\breve{\mathbf{a}}-\mathbf{a}_{0}\right\|^{2}-\epsilon\right)
$$

where $\lambda \geq 0$ is the Lagrange multiplier. The estimae of $\breve{a}$ is obtained as [7]

$$
\widehat{\breve{a}}=\mathbf{a}_{0}-\mathbf{U}(\mathbf{I}+\lambda \boldsymbol{\Lambda})^{-1} \mathbf{U}^{H} \mathbf{a}_{0},
$$

where

$$
\widehat{\mathbf{R}}_{\mathrm{fs}}=\mathbf{U} \boldsymbol{\Lambda} \mathbf{U}^{H},
$$

with the columns in $\mathbf{U}$ denoting the eigenvectors of $\hat{\mathbf{R}}_{\mathrm{fs}}$ and the diagonal elements of the diagonal matrix $\Lambda$ the corresponding eigenvalues of $\hat{\mathbf{R}}_{\mathrm{fs}}$.

Once the estimate of $\breve{a}$ is obtained, a data-dependent weight vector $\hat{\mathbf{g}}$ can be determined by [7]

$$
\begin{aligned}
\hat{\mathbf{g}} & =\frac{\hat{\mathbf{R}}_{\mathrm{fs}}^{-1} \hat{\mathbf{a}}}{\widehat{\hat{\mathbf{a}}}^{H} \widehat{\mathbf{R}}_{\mathrm{fs}}^{-1} \hat{\mathbf{a}}} \\
& =\frac{\left(\widehat{\mathbf{R}}_{\mathrm{fs}}+(1 / \lambda) \mathbf{I}\right)^{-1} \mathbf{a}_{0}}{\mathbf{a}_{0}^{H}\left(\hat{\mathbf{R}}_{\mathrm{fs}}+(1 / \lambda) \mathbf{I}\right)^{-1} \widehat{\mathbf{R}}_{\mathrm{fs}}\left(\widehat{\mathbf{R}}_{\mathrm{fs}}+(1 / \lambda) \mathbf{I}\right)^{-1} \mathbf{a}_{0}} .
\end{aligned}
$$

Note that $\hat{\mathbf{g}}$ is a vector of length $M_{1} M_{2}$. Let $\widehat{\mathbf{G}}$ be a $2 \mathrm{D}$ $M_{1} \times M_{2}$ matrix obtained by reshaping $\hat{\mathbf{g}}$ by using the first $M_{1}$ elements of $\hat{\mathbf{g}}$ as the first column of $\hat{\mathbf{G}}$ and so forth. Then $\widehat{\mathbf{G}}$ is the impulse response of a $2 \mathrm{D}$ finite impulse response (FIR) filter with taps $M_{1}$ and $M_{2}$ in the fast- and slow-time dimensions, respectively. The 2D FIR filter is applied to each $2 \mathrm{D}$ data matrix $\mathbf{X}_{n_{\mathrm{c}}, n_{\mathrm{p}}}$. The 2D FIR filter output matrix $\mathbf{Y}_{n_{\mathrm{c}}, n_{\mathrm{p}}}$, corresponding to $\mathbf{X}_{n_{\mathrm{c}}, n_{\mathrm{p}}}$, has dimension $L_{1} \times L_{2}$.

By stacking the 2D FIR filter output matrices $\left\{\mathbf{Y}_{n_{\mathrm{c}}, n_{\mathrm{p}}}\right\}_{n_{\mathrm{c}}=0}^{N_{\mathrm{c}}}$ from each antenna on top of each other and including all $N_{\mathrm{p}}$ data sets, at each scan location, we obtain a $2 \mathrm{D}$ complexvalued data matrix of dimension $\left(N_{\mathrm{c}}+1\right) L_{1} \times N_{\mathrm{p}} L_{2}$. The data vector $\mathbf{y}_{l}$ consisting of the $2 \mathrm{D}$ FIR filter output samples of all antennas at the $l$ th echo has the form

$$
\mathbf{y}_{l}=\beta \overline{\mathbf{a}} \bar{s}_{l}+\overline{\mathbf{e}}_{l}, \quad l=1, \ldots, N_{\mathrm{p}} L_{2},
$$

where $\beta$ has the same meaning as in (2), $\overline{\mathbf{a}}$ acts as a steering vector similar to a in (2) but with a longer length $\left(N_{\mathrm{c}}+1\right) L_{1}$ (since $L_{1}$ can be much larger than $\left.N_{\mathrm{b}}\right), \bar{s}_{l}$ is the signal waveform given by $\bar{s}_{l}=\gamma\left(\bmod \left[l-1, L_{2}\right]+1\right), l=1, \ldots, N_{\mathrm{p}} L_{2}$, $\overline{\mathbf{e}}_{l}$ is a vector containing the $2 \mathrm{D}$ FIR filter output due to the RFIs and noise associated with the $l$ th echo, which we assume to be spatially colored but temporally white (due to the $2 \mathrm{D}$ FIR filtering in the fast- and slow-time dimensions) Gaussian with unknown and arbitrary, but fixed, spatial covariance matrix. The total number of snapshots associated with the data model in (32) is $N_{\mathrm{p}} L_{2}$.

The data model in (32) accounts for the joint fast-time and spatial data information. The steering vector $\overline{\mathbf{a}}$ contains the fast-time responses of the QR signal at all antennas (spatial channels). The first $L_{1}$ elements of $\overline{\mathbf{a}}$ correspond to the main antenna and are ones (similar to (25), this is an approximation since the QR signal is not a constant strictly over the fast time) and the remaining $N_{\mathrm{c}} L_{1}$ elements of $\overline{\mathbf{a}}$ correspond to the reference antennas and are zeros.

Due to the similarity between the two data models in (2) and (32), the spatial ML approach devised in Section 3.1 and based on the data model in (2) can be directly applied to the 2D FIR filter output data (modeled in (32)) to obtain the ML estimate of the QR signal amplitude.

However, because the data model in (32) accounts for both fast-time and spatial data information, the size of the joint fast-time and spatial dimension can be very large. To reduce the dimension by half, let $\mathbf{Y}_{n_{\mathrm{c}}, n_{\mathrm{p}}, 1}$ and $\mathbf{Y}_{n_{\mathrm{c}}, n_{\mathrm{p}}, 2}$ be two submatrices of $\mathbf{Y}_{n_{\mathrm{c}}, n_{\mathrm{p}}}$ containing the first and last (1/2) $L_{1}\left(L_{1}\right.$ needs to be an even number) rows of $\mathbf{Y}_{n_{\mathrm{c}}, n_{\mathrm{p}}}$, respectively. Let $\mathbf{Z}_{n_{\mathrm{c}}, n_{\mathrm{p}}}=\left[\mathbf{Y}_{n_{\mathrm{c}}, n_{\mathrm{p}}, 1} \mathbf{Y}_{n_{\mathrm{c}}, n_{\mathrm{p}}, 2}\right]$. We stack the columns of the matrices $\left\{\mathbf{Z}_{n_{\mathrm{c}}, n_{\mathrm{p}}}\right\}_{n_{\mathrm{c}}=0}^{N_{\mathrm{c}}}$ on top of each other and include all such $N_{\mathrm{p}}$ data matrices. Then we obtain a $2 \mathrm{D}$ data matrix of dimension $(1 / 2)\left(N_{\mathrm{c}}+1\right) L_{1} \times 2 N_{\mathrm{p}} L_{2}$ at each scan location. With this rearrangement, the size of the joint fast-time and spatial dimension is reduced by half while the number of the snapshots is doubled.

Note that the structure of the joint fast-time and spatial data model in (32) is still valid for the rearranged matrix above. Therefore, we can directly apply the ML approach to the rearranged data to estimate the QR signal amplitude. For notational convenience, we continue to use the same notations as in (32) for the rearranged matrix. The steering vector $\overline{\mathbf{a}}$ needed to apply the ML estimator now consists of $(1 / 2)\left(N_{c}+1\right) L_{1}$ elements with the first $(1 / 2) L_{1}$ elements corresponding to the main antenna and being ones and with all the remaining $(1 / 2) N_{\mathrm{c}} L_{1}$ elements corresponding to the reference antennas and being zeros. The QR signal waveform for the rearranged 2D FIR filter output data is $\bar{s}_{l}=\gamma\left(\bmod \left[l-1, L_{2}\right]+1\right), l=1, \ldots, 2 N_{\mathrm{p}} L_{2}$.

We refer to the RFI mitigation via the joint fast- and slowtime 2D RCB and joint fast-time and spatial ML approach introduced in this subsection as Method 3, which includes the following steps.

Step 1. Estimating the 2D FIR filter by using 2D RCB jointly in the fast- and slow-time dimensions.

Step 2. Using the 2D FIR filter to mitigate the RFIs jointly in the fast- and slow-time dimensions and rearranging the output data.

Step 3. Applying the joint fast-time and spatial ML estimator to the rearranged 2D FIR filter output data to obtain the ML estimate of the QR signal amplitude.

Step 4. Calculating the output SNR.

\subsection{Combined approach for RFI mitigation}

We may combine the merits of all three aforementioned approaches for TNT detection. The combined approach is a three-stage detector as shown in Figure 4.

We first use Method 1 as a baseline and then progressively employ Methods 2 and 3. At a scan location, if the estimated 


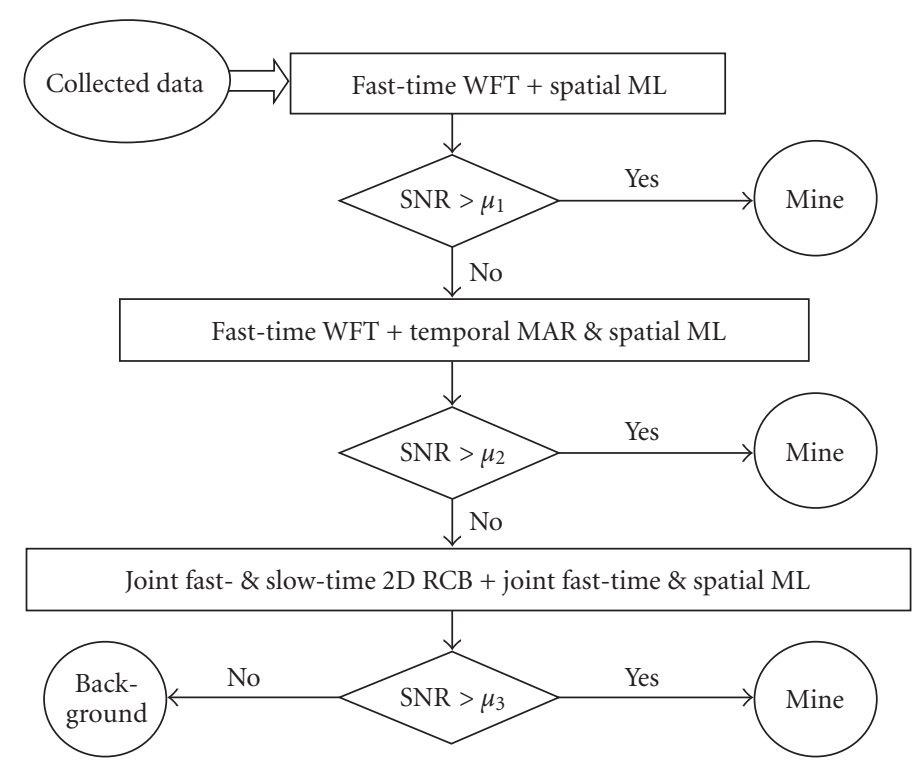

FIgure 4: Combined detector for TNT detection by QR.

TABLE 1: Description of the three experimental data sets.

\begin{tabular}{ccccc}
\hline Dataset & $\begin{array}{c}\text { Number of } \\
\text { mine scans }\end{array}$ & $\begin{array}{c}\text { Number of } \\
\text { background scans }\end{array}$ & $\begin{array}{c}\text { Number of antennas } \\
\text { used }\left(N_{\mathrm{c}}+1\right)\end{array}$ & $\begin{array}{c}\text { Number of TNT files } \\
\text { after deringing }\left(N_{\mathrm{p}}\right)\end{array}$ \\
\hline 1 & 90 & 90 & 4 & 5 \\
2 & 100 & 160 & 4 & 5 \\
3 & 178 & 160 & 4 & 5 \\
\hline
\end{tabular}

SNR via Method 1 is greater than a prespecified threshold (say $\mu_{1}$ ), we claim that there is a mine at this scan location. Otherwise, the detector goes on and uses Method 2 for making a decision. If the estimated SNR via Method 2 is greater than the second prespecified threshold ( $\operatorname{say} \mu_{2}$ ), we claim the presence of the mine. If not, Method 3 is then applied. Again, the estimated SNR is compared with a detection threshold $\left(\right.$ say $\left.\mu_{3}\right)$ and a final decision is made.

\section{EXPERIMENTAL RESULTS}

We present experimental results to illustrate the performance of the proposed approaches for landmine detection by QR. Three data sets (referred to as Datasets 1, 2, and 3, resp.) collected by the QR sensor built by QM are used in our experimental examples. Descriptions of the data sets are listed in Table 1.

Dataset 1 contains 90 scans from a TNT simulant and 90 scans from background. Dataset 2 contains 100 and 160 scans from mines and background, respectively. Of the 100 mine scans in Dataset 2, 40 TNT mines are buried at $5^{\prime \prime}$ depth, and 60 TNT mines at $3^{\prime \prime}$ depth. Both types of mines are plastic-cased. There are 178 mine scans (for a plastic-cased TNT mine) and 160 background scans in Dataset 3. When the data were collected, the QR sequence was automatically optimized based on the estimated mine temperature entered by the system operator. For all the data sets, $N_{\mathrm{f}}=50, N_{\mathrm{s}}=54$, $T_{\mathrm{f}}=10^{-5}$ seconds, and $T_{\mathrm{s}}=1.15 \times 10^{-3}$ seconds. After deringing, there are $N_{\mathrm{p}}=5,13$, and 5 TNT files left for each scan in Datasets 1, 2, and 3, respectively. Each TNT file corresponds to a pulse loop. For each scan, we exploit data samples from 4 antennas ( 1 main and 3 reference antennas).

It is observed that strong RFIs appear just around the QR signal frequency for Dataset 3. Figure 5 shows an example of a $2 \mathrm{D}$ fast- and slow-time data matrix $($ size $50 \times 54)$ collected by the main antenna over a mine in Dataset 3. Figure 5(a) is the time-domain image (real part) of the $2 \mathrm{D}$ data matrix where the horizontal and vertical axes are for the slow- and fast-time sample indices, respectively, Figures 5(b) and 5(c) are the magnitudes of the 1D FT images obtained by performing 1D FT along the slow- and fast-time dimensions of the $2 \mathrm{D}$ data matrix, respectively, and Figure $5(\mathrm{~d})$ is the magnitude image of the 2D FT of the 2D data matrix. The horizontal axis of Figures 5(b) and 5(d) is for the slow frequency normalized with the slow-time sampling frequency $1 / T_{s}$. The vertical axis of Figures 5(c) and 5(d) is for the fast frequency normalized with the fast-time sampling frequency $1 / T_{\mathrm{f}}$. The center of the image in Figure 5(d) (marked with a circle) is the frequency location of the QR signal. From Figure 5(c), it is clear that one of the strong RFI carrier frequencies is very 


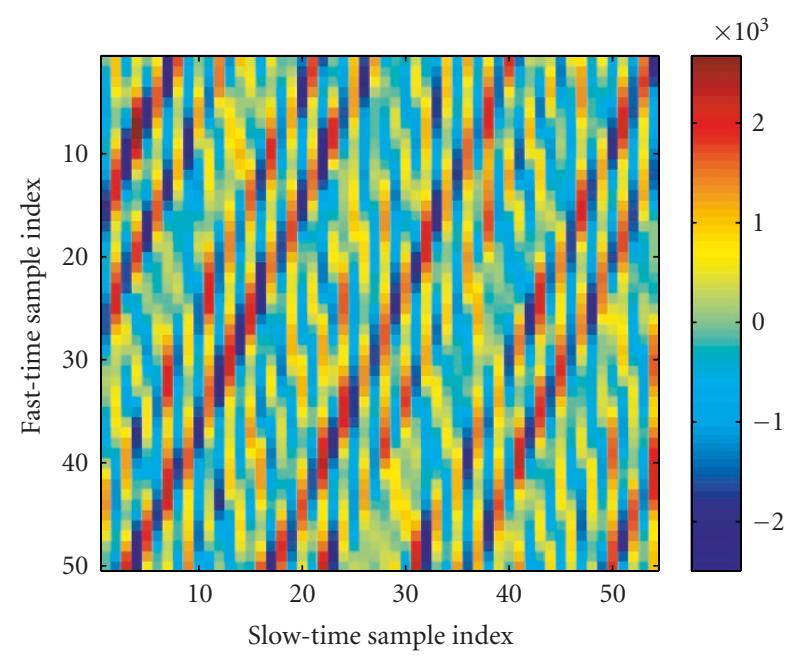

(a)

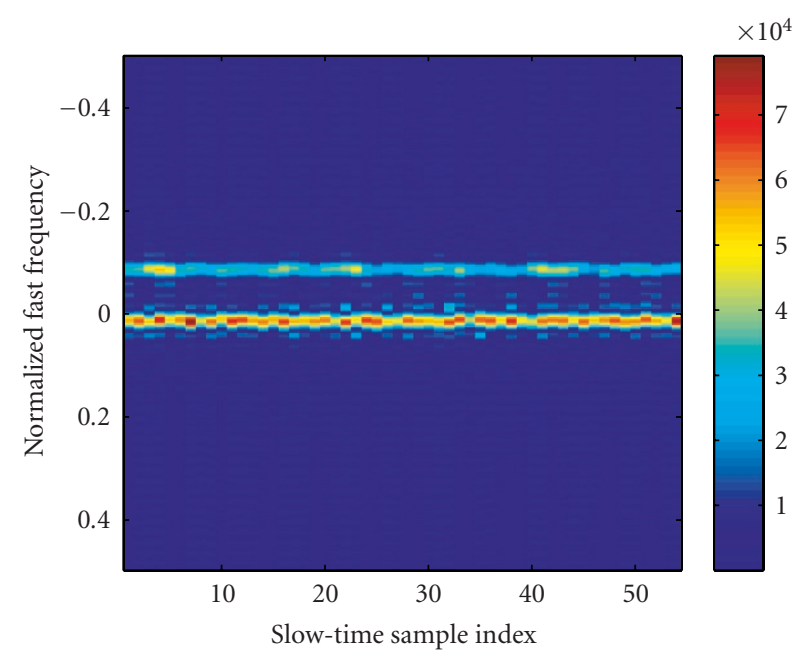

(c)

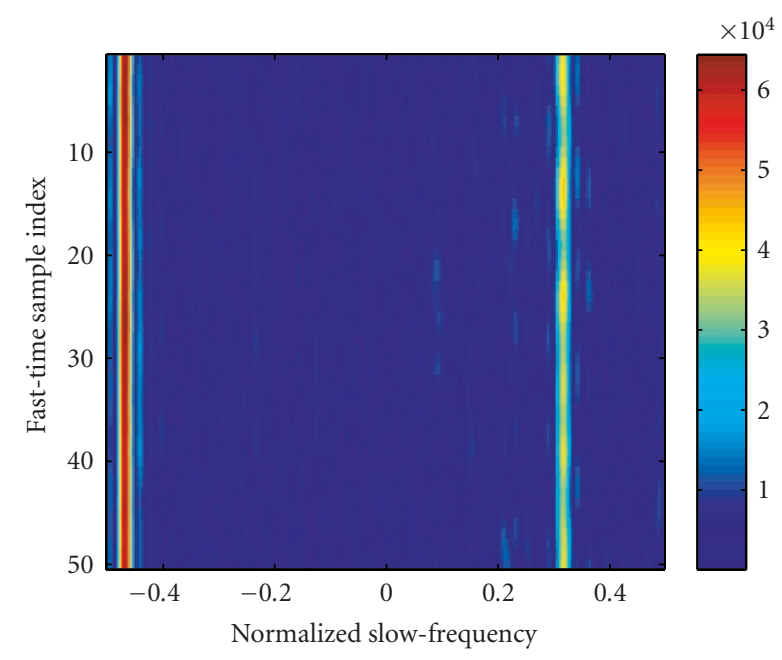

(b)

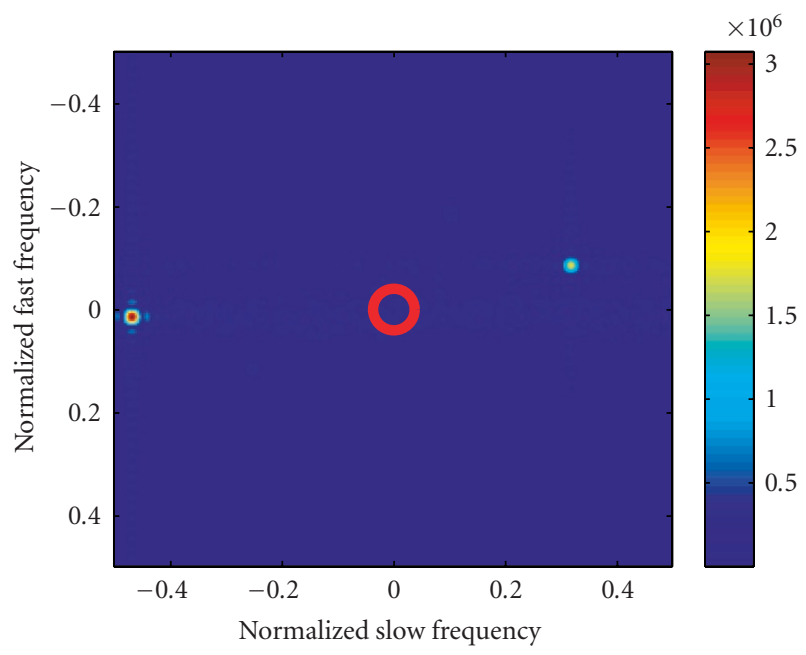

(d)

FIGURE 5: Time and frequency images of data samples received by the main antenna over a mine; (a) 2D time-domain image (real part), (b) magnitude of FFT image along slow-time dimension, (c) magnitude of FFT image along fast-time dimension, and (d) magnitude of 2D FFT image (the center of the circle is the zero-frequency location of the QR signal, which is too weak to be seen).

close to the QR signal frequency (in the middle or around zero) in the fast-frequency dimension. This shows the challenge of the QR signal detection in the presence of strong RFIs. From Figure 5(b), we note that the RFIs are not white in the slow-time dimension and their carrier frequencies are far from the QR signal frequency (in the middle or around zero) in the slow-frequency dimension. This figure verifies the motivation to mitigate the RFIs by exploiting the temporal (slow-time) correlation of the RFIs.

For Methods 1 and 2, we use a Hanning window in the fast-time dimension prior to performing FT and $N_{\mathrm{b}}=3$ frequency bins are picked up from each reference channel. We choose $K=1$ (i.e., first order) for the MAR filtering for Method 2. Regarding the implementation of 2D RCB for Method 3, we choose the numbers of taps $M_{1}=19$ and $M_{2}=2$ in the fast- and slow-time dimensions, respectively, and $\epsilon=0.1$ is adopted for RCB to allow uncertainty for the steering vector. As for the combined approach, we choose $\mu_{1}=2.2$ and $\mu_{2}=1.7$ as the thresholds to activate Methods 2 and 3 , respectively. We then change the third threshold $\mu_{3}$ to obtain a series of values of probability of detection $(\mathrm{Pd})$ versus false alarm rate (FAR), which form the receiver operating characteristic (ROC) curve as a performance indicator. For a given threshold, $\mathrm{Pd}$ is given by the ratio of the number of detected mine scans over the number of total mine scans, and FAR is the ratio of the number of background scans whose estimated SNR values exceed the threshold over the number of total background scans.

First, we present an example to demonstrate how our proposed methods are used to mitigate the RFIs by exploiting both the spatial and temporal correlations of the RFIs. In this example, we apply the 2D RCB approach involved in 


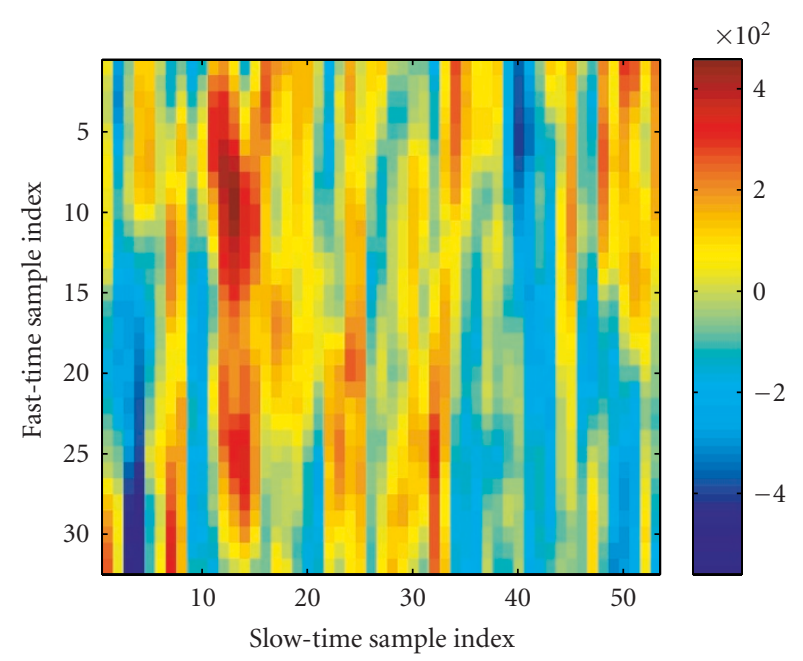

(a)

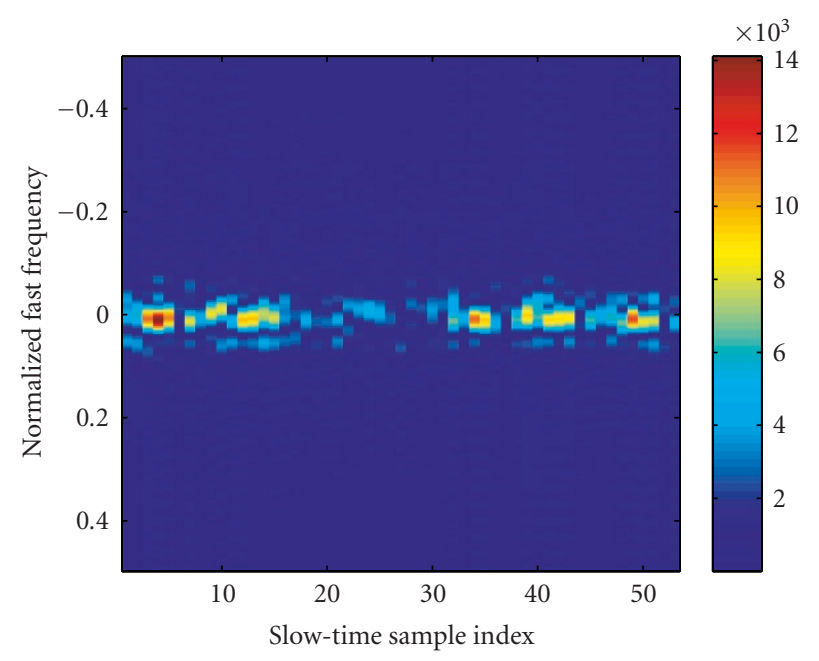

(c)

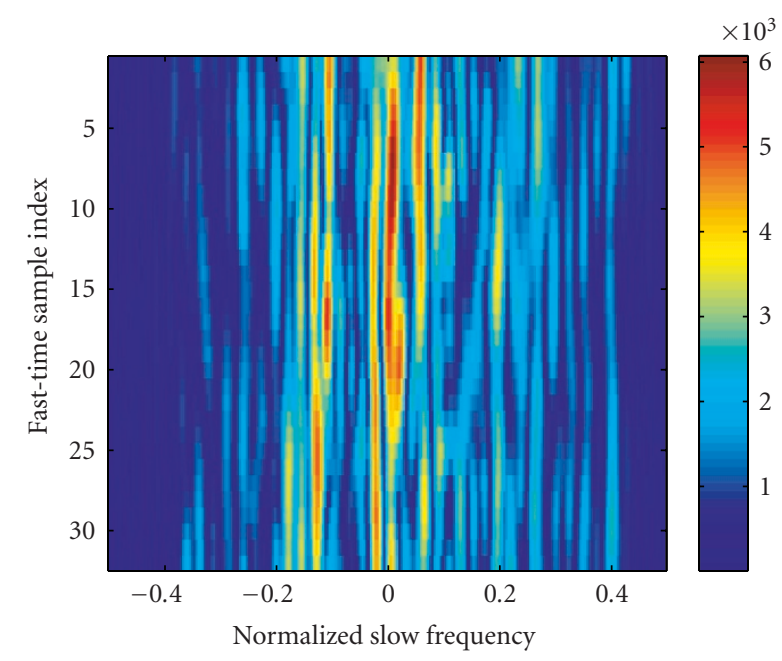

(b)

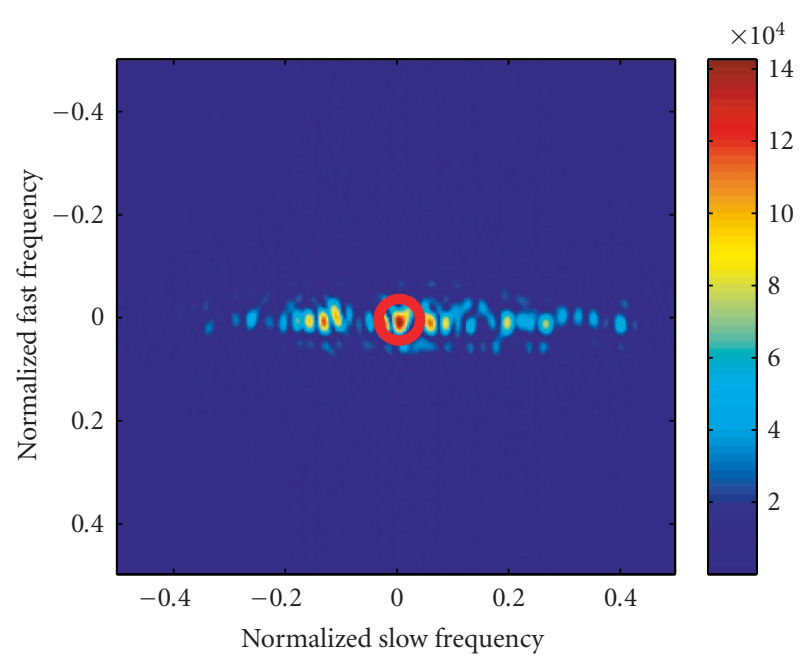

(d)

FIgURE 6: Time and frequency images after RFI mitigation via 2D RCB for the data samples considered in Figure 5; (a) 2D time-domain image (real part), (b) magnitude of FFT image along slow-time dimension, (c) magnitude of FFT image along fast-time dimension, and (d) magnitude of 2D FFT image (the center of the circle is the zero-frequency location of the QR signal, which can be clearly seen after the RFI mitigation).

Method 3 to the QR data collected from the mine considered in Figure 5. For the 2D RCB filtered data, Figures 6(a) through 6(d) show the time and frequency images that correspond to Figures $5(\mathrm{a})$ through 5(d), respectively. We see from Figures 6(b) and 6(c) that the strong RFIs have been mitigated by the 2D RCB filtering, and as a result in the center of the image in Figure 6(d), the QR signal clearly shows up. Also note from Figure 6(d) that there are still some residual interference components remaining around the zero-frequency component. These residues are to be reduced by exploiting the spatial information in the ML processing step of Method 3.

In this example, we apply both Methods 1 and 2 to the same mine scan considered in Figures 5 and 6. Figure 7(a) plots the middle row (corresponding to the zero fastfrequency bin) of Figure 5(d), which is the spectral pattern of the zero-frequency samples from all the $N_{\mathrm{s}}$ acquisition windows in the first TNT file for the main antenna. Once again, the dominating RFI indicates that the RFI is temporally colored. Figure 7(b) presents the output of the filtering in the spatial domain by Method 1 according to (10), while Figure 7(c) gives the corresponding result by using Method 2. It is clear that the only spatial ML approach itself does not produce a satisfactory RFI mitigation result. Note the significant improvement in the RFI mitigation achieved by using Method 2. Figure 7(d) presents the result of the filtering in the spatial domain produced by Method 3. We note that Method 3 outperforms Method 2 in that the former produces 


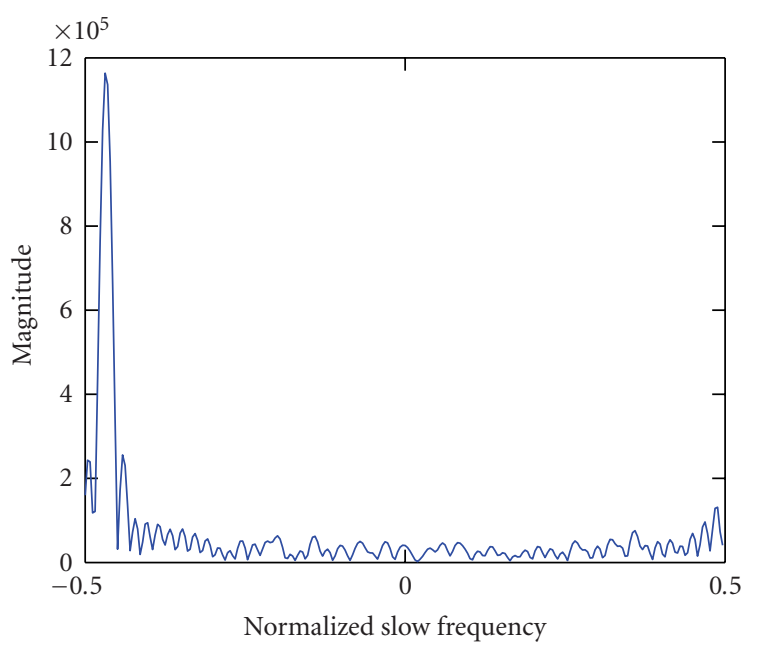

(a)

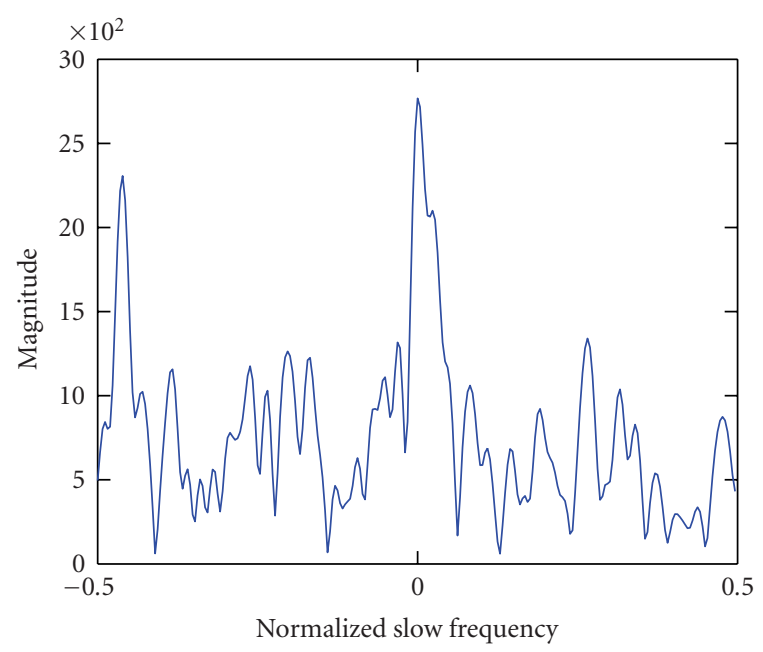

(c)

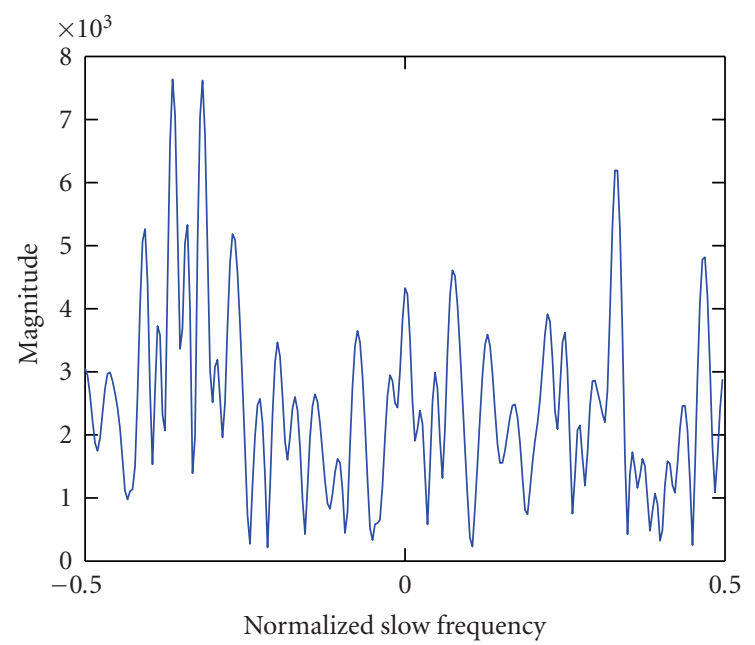

(b)

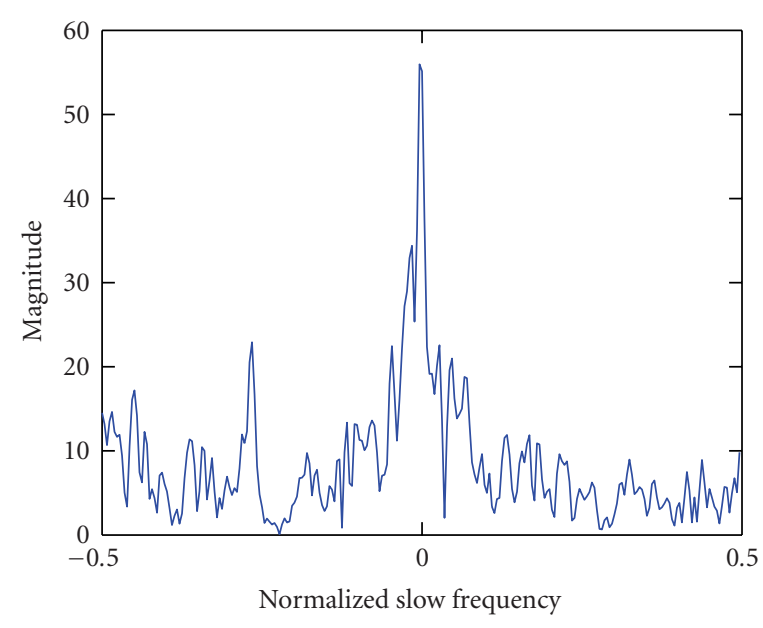

(d)

FIGURE 7: RFI mitigation example for the mine considered in Figure 5; (a) magnitude of the spectrum of the zero-frequency sampling sequence from the main antenna, (b) filtering output in the spatial domain by Method 1, (c) filtering output in the spatial domain by Method 2, and (d) filtering output in the spatial domain by Method 3.

narrower main beamwidth and lower residual interference and noise spectra than the latter.

Next, we examine the effects of the snapshot doubling manipulation discussed in Section 3.3 on the detection performance. We apply Method 3 to Dataset 3 in two cases, with and without snapshot doubling. The attained ROC curves are plotted in Figure 8, from which we see that the snapshot doubling does help improving the detection performance. The performance of using $N_{\mathrm{p}}=4$ TNT files with snapshot doubling is comparable to that of using $N_{\mathrm{p}}=5$ TNT files without snapshot doubling. This demonstrates the usefulness of the snapshot doubling manipulation.

We now compare the RFI mitigation performance via various processing schemes. Particularly, we compare our proposed data-adaptive approaches, Methods 1, 2, and 3, with the data-independent DAS approach and the adaptive
SCB approach. The implementation of the DAS approach is similar to that of Method 1 with the matrix $\mathbf{T}$ in (9) being replaced by an identity matrix. The steps of 2D SCB follow those of 2D RCB as introduced in Section 3.3 and Figure 2 except that SCB replaces RCB. Similar to Method 3, the 2D SCB approach is followed by the spatial ML approach based on the data model in (32). We show in Figure 9 the ROC curves of applying the five approaches to Dataset 3. As expected, all the adaptive approaches significantly outperform the nonadaptive DAS approach. We note from Figure 9 that 2D RCB performs better than 2D SCB.

Finally, we apply our proposed methods to all three data sets. Figures 10(a) through 10(c) present the ROC curves for Method 1 (dashed and dotted line), Method 2 (dotted line), Method 3 (dashed line), and the combined approach (solid line) when using Datasets 1, 2, and 3, respectively. The RFIs 


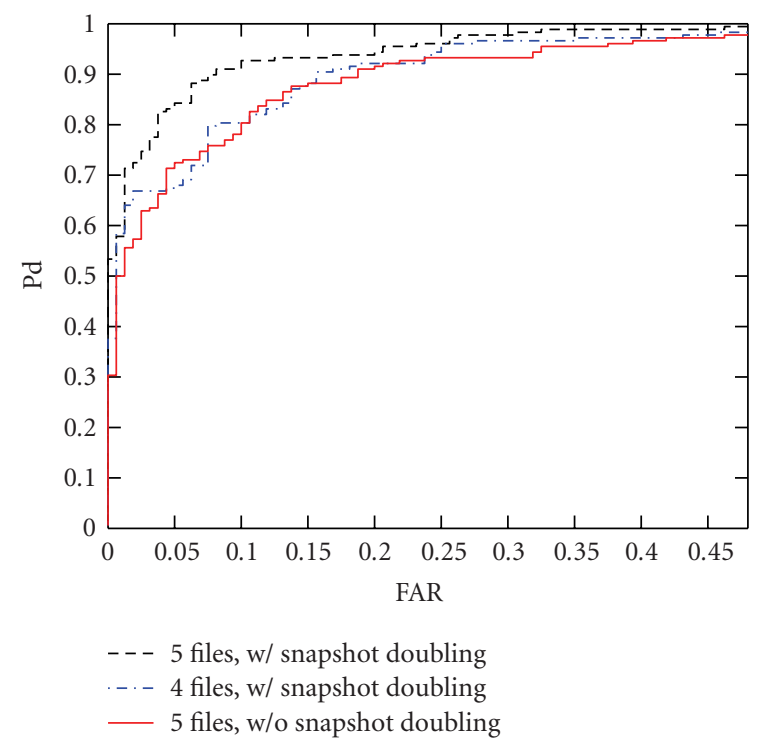

FIGURE 8: Detection performance comparison between with and without the snapshot doubling manipulation for Method 3 when applied to Dataset 3.

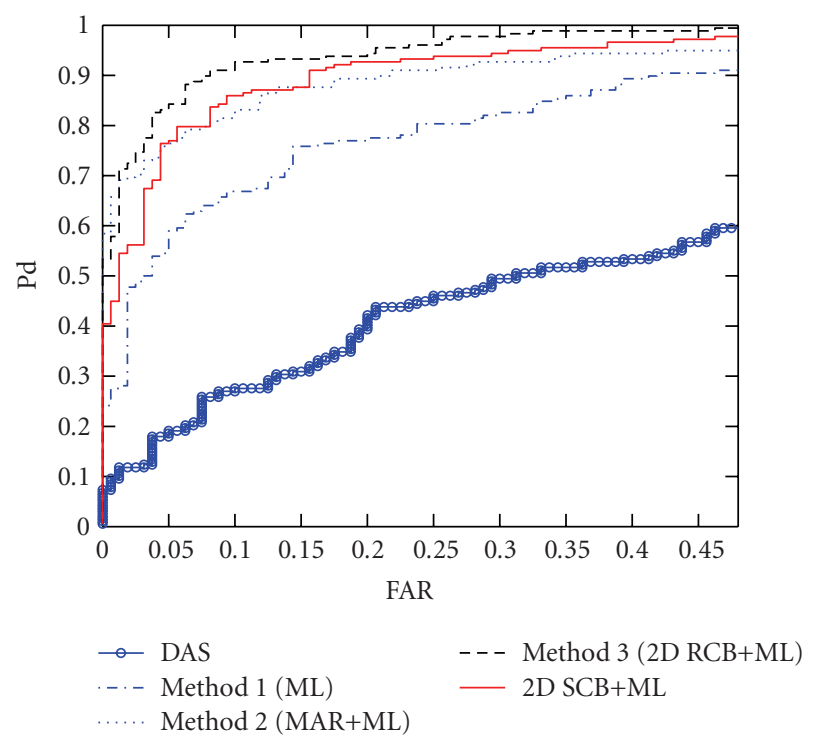

FIGURE 9: Detection performance comparison of DAS (solid line with $\circ$ ), Method 1 (dashed and dotted line), Method 2 (dotted), Method 3 (dashed line), and 2D SCB followed by the spatial ML approach (solid line).

in Dataset 1 are not close to the QR signal frequency and Method 1 works better than Methods 2 and 3 for Dataset 1. Method 3 performs consistently and performs much better than Methods 1 and 2 for Datasets 2 and 3, where an RFI is very close to the QR signal frequency (this is especially so for Dataset 3). Figure 10(d) presents the ROC curves for the case of using all three data sets. Overall, these results in Figure 10 verify the necessity of taking into account the temporal correlation of RFIs when performing RFI mitigation and demonstrate the effectiveness of our RFI mitigation approaches that exploit both the spatial and temporal correlations of RFIs. The robustness of our combined approach is demonstrated as well due to using data sets collected at different times and conditions.

\section{CONCLUSIONS}

We have investigated the RFI mitigation for landmine detection by QR. We have exploited both the spatial and temporal correlations of the RFIs to improve the TNT detection performance. We have first considered exploiting the spatial correlations of the RFIs only and proposed an ML estimator for signal amplitude estimation and a CFAR detector for TNT detection. We have then adopted an MAR model to take into account the temporal correlation of the RFIs for RFI mitigation. We have also considered using joint fast- and slow-time 2D RCB and joint fast-time and spatial ML estimator for RFI mitigation. Finally, we have combined the merits of all three methods for TNT detection. The experimental results with three data sets collected by Quantum Magnetics (QM), Inc., have been used to verify the necessity of taking into account the temporal correlation of RFIs when performing RFI mitigation and demonstrated the effectiveness of our RFI mitigation approaches that exploit both the spatial and temporal correlations of RFIs. The combined approach has also been shown to outperform all the three proposed methods, and its robustness has been demonstrated as well due to using data sets collected at different times and conditions.

\section{APPENDIX}

\section{CFAR PROPERTY OF (13)}

We now show that the statistic defined in (13) is independent of the noise and interference scenario, and thus it is a CFAR test. To facilitate our discussion, we recall (6),

$$
\mathbf{x}_{\mathrm{s}}=\frac{1}{L} \sum_{l=1}^{L} \mathbf{x}_{l} s_{l}^{*} .
$$

A straightforward calculation shows that $\mathbf{x}_{\mathrm{s}} \sim N\left(P_{\mathrm{s}} \beta \mathbf{a}\right.$, $(P s / L) \mathbf{Q})$. Denoting that $\chi=\sqrt{\left(L / P_{s}\right)} \mathbf{x}_{s}$, we have

$$
\mathrm{SNR}=\frac{\left[\operatorname{Re}\left(\mathbf{a}^{H} \mathbf{T}^{-1} \chi\right)\right]_{+}^{2}}{\mathbf{a}^{H} \mathbf{T}^{-1} \mathbf{a}+(1 / L) \operatorname{Im}^{2}\left(\mathbf{a}^{H} \mathbf{T}^{-1} \chi\right)},
$$

where $\chi \sim N\left(\sqrt{L P_{s}} \beta \mathbf{a}, \mathbf{Q}\right)$. Furthermore, letting

$$
\begin{aligned}
\mathbf{C} & =\mathbf{Q}^{-1 / 2} \mathbf{T} \mathbf{Q}^{-1 / 2}, \\
\mathbf{d} & =\mathbf{Q}^{-1 / 2} \mathbf{a}, \\
\zeta & =\mathbf{Q}^{-1 / 2} \chi,
\end{aligned}
$$

we have $\mathbf{C} \sim C W(M, L-1 ; \mathbf{I})$ with $M$ denoting the length of a and $\mathbf{I}$ being an identity matrix, which is a complex Wishart distribution, and $\zeta \sim N(0, \mathbf{I})$ when no target is present. It follows from the transformation defined in (.35) that

$$
\mathrm{SNR}=\frac{\left[\operatorname{Re}\left(\mathbf{d}^{H} \mathbf{C}^{-1} \zeta\right)\right]_{+}^{2}}{\mathbf{d}^{H} \mathbf{C}^{-1} \mathbf{d}+(1 / L) \operatorname{Im}^{2}\left(\mathbf{d}^{H} \mathbf{C}^{-1} \zeta\right)}
$$




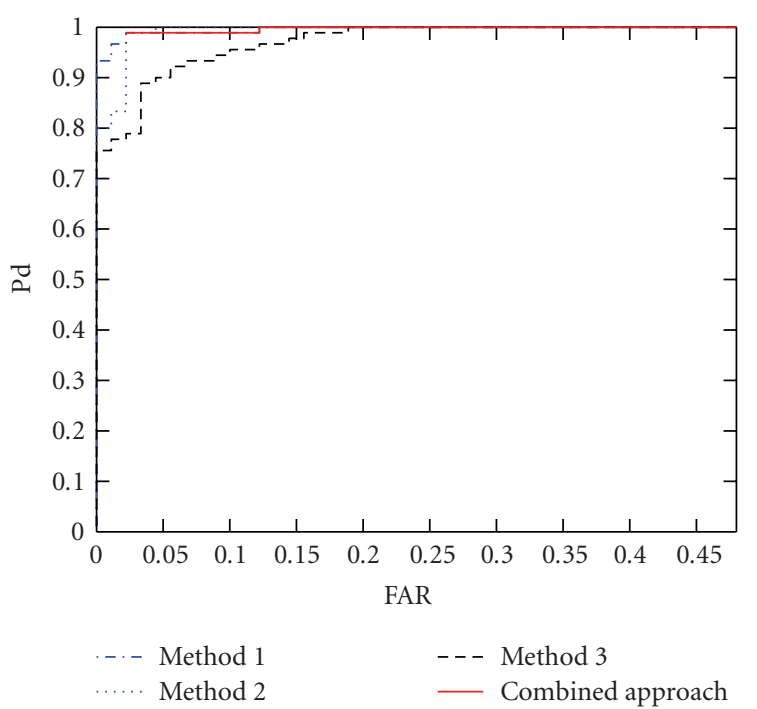

(a)

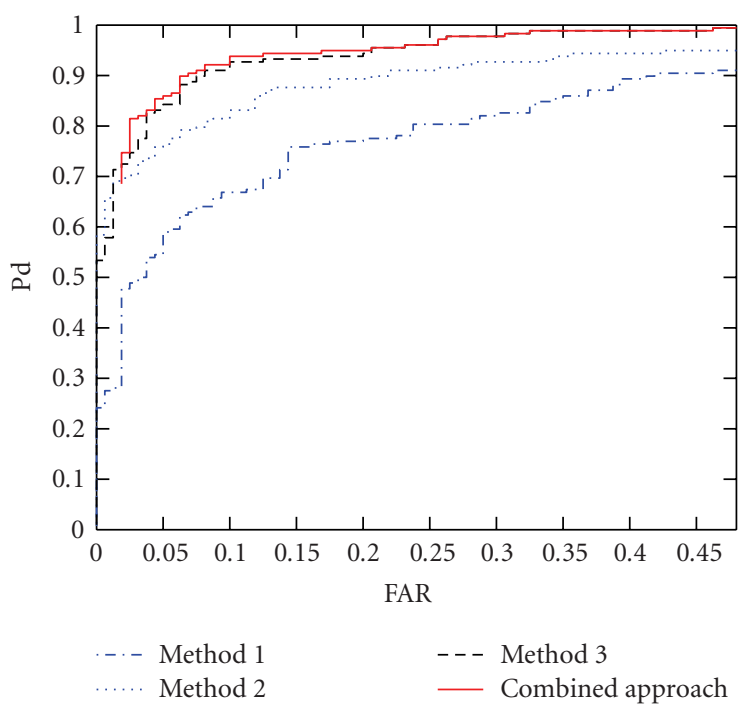

(c)

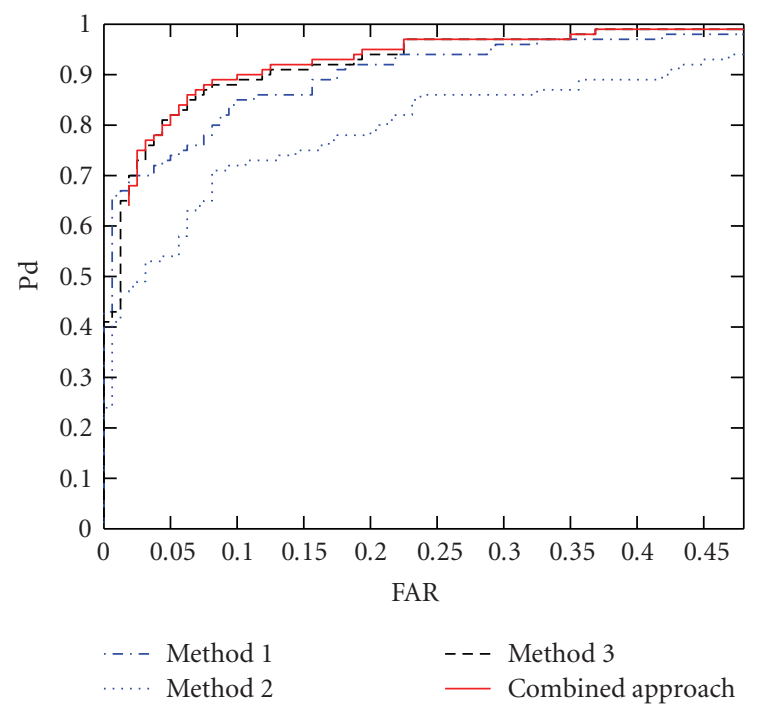

(b)

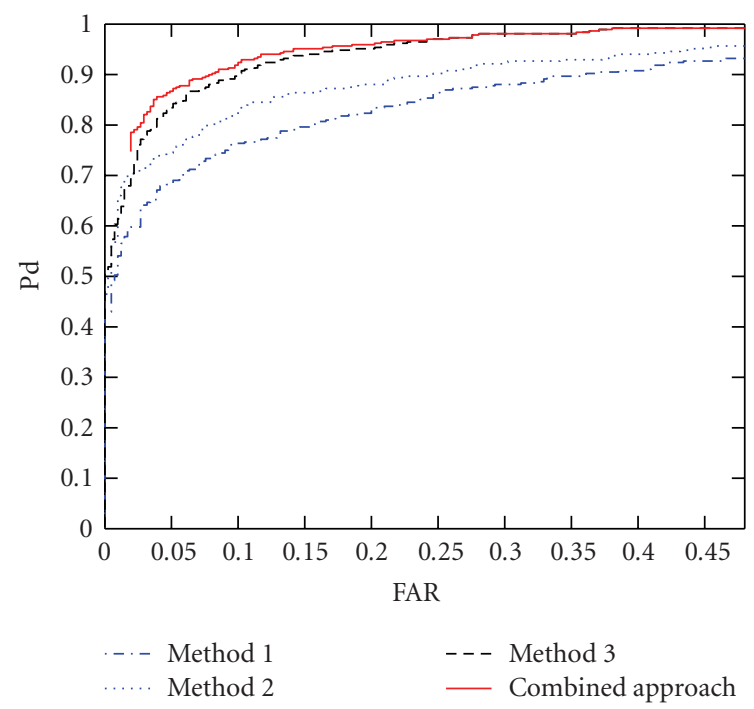

(d)

FIGURE 10: Detection performance of Method 1 (dashed and dotted line), Method 2 (dotted line), Method 3 (dashed line), and the combined approach (solid line) for (a) Dataset 1, (b) Dataset 2, (c) Dataset 3, and (d) all three data sets together.

Note that the random variables $\mathbf{C}$ and $\zeta$ are both invariant to a unitary transformation. We can design a unitary matrix $\mathbf{V}$ such that

$$
\begin{aligned}
\mathbf{V}^{H} \mathbf{d} & =\|\mathbf{d}\|\left[\begin{array}{ll}
1 & \mathbf{0}_{M-1}^{T}
\end{array}\right]^{T}, \\
& \triangleq\|\mathbf{d}\| \mathcal{E},
\end{aligned}
$$

where $\mathbf{0}_{M-1}$ is an all-zero vector of length $M-1,\|\cdot\|$ denotes the Euclidean norm, and $(\cdot)^{H}$ and $(\cdot)^{T}$ denote the conjugate transpose and the transpose, respectively. Thus (.36) can be simplified as

$$
\mathrm{SNR}=\frac{\left[\operatorname{Re}\left(\varepsilon^{H} \mathbf{D}^{-1} \boldsymbol{\eta}\right)\right]_{+}^{2}}{\varepsilon^{H} \mathbf{D}^{-1} \varepsilon+(1 / L) \operatorname{Im}^{2}\left(\varepsilon^{H} \mathbf{D}^{-1} \boldsymbol{\eta}\right)},
$$

where $\mathbf{D}=\mathbf{V}^{H} \mathbf{C V}$, and $\boldsymbol{\eta}=\mathbf{V}^{H} \zeta$. It is clear from (.38) that the SNR is independent of the interference-and-noise scenario. Thus the proposed statistic is a CFAR test.

\section{ACKNOWLEDGMENTS}

This work was supported in part by the US Army under Contract DAAB15-01D-0004 and the National Science Foundation Grant CCR-0104887.

\section{REFERENCES}

[1] A. N. Garroway, M. L. Buess, J. B. Miller, et al., "Remote sensing by nuclear quadrupole resonance," IEEE Transactions on Geoscience and Remote Sensing, vol. 39, no. 6, pp. 1108-1118, 2001. 
[2] A. D. Hibbs, G. A. Barrall, P. V. Czipott, et al., "Land mine detection by nuclear quadrupole resonance," in Detection and Remediation Technologies for Mines and Minelike Targets III, vol. 3392 of Proceedings of SPIE, pp. 522-532, Orlando, Fla, USA, April 1998.

[3] A. D. Hibbs, G. A. Barrall, P. V. Czipott, et al., "Detection of TNT and RDX landmines by standoff nuclear quadrupole resonance," in Detection and Remediation Technologies for Mines and Minelike Targets IV, vol. 3710 of Proceedings of SPIE, pp. 454-463, Orlando, Fla, USA, April 1999.

[4] R. M. Deas, I. A. Burch, and D. M. Port, "Detection of RDX and TNT mine-like targets by nuclear quadrupole resonance," in Detection and Remediation Technologies for Mines and Minelike Targets VII, vol. 4742 of Proceedings of SPIE, pp. 482-489, Orlando, Fla, USA, April 2002.

[5] Y. Jiang, P. Stoica, and J. Li, "Array signal processing in the known waveform and steering vector case," IEEE Transactions on Signal Processing, vol. 52, no. 1, pp. 23-35, 2004.

[6] A. L. Swindlehurst and P. Stoica, "Maximum likelihood methods in radar array signal processing," Proceedings of the IEEE, vol. 86, no. 2, pp. 421-441, 1998.

[7] J. Li, P. Stoica, and Z. Wang, "On robust Capon beamforming and diagonal loading," IEEE Transactions on Signal Processing, vol. 51, no. 7, pp. 1702-1715, 2003.

[8] J. Ward, "Space-time adaptive processing for airborne radar," Tech. Rep. 1015, MIT Lincoln Laboratory, Lexington, Mass, USA, December 1994.

[9] T. Söderström and P. Stoica, System Identification, PrenticeHall, London, UK, 1989.

[10] J. Capon, "High-resolution frequency-wavenumber spectrum analysis," Proceedings of the IEEE, vol. 57, no. 8, pp. 1408-1418, 1969.

[11] H. L. Van Trees, Optimum Array Processing: Part IV of Detection, Estimation, and Modulation Theory, John Wiley \& Sons, New York, NY, USA, 2002.

[12] D. D. Feldman and L. J. Griffiths, "A projection approach for robust adaptive beamforming," IEEE Transactions on Signal Processing, vol. 42, no. 4, pp. 867-876, 1994.

[13] P. Stoica, Z. Wang, and J. Li, "Robust Capon beamforming," IEEE Signal Processing Letters, vol. 10, no. 6, pp. 172-175, 2003.

Guoqing Liu received the B.S., M.S., and Ph.D. degrees in electrical engineering from University of Electronic Science and Technology of China, Chengdu, China, in 1988, 1991, and 1996, respectively. From August 1993 to December 1995, he was a Visiting Scholar at the Department of Remote Sensing Applications, Alenia Spazio SPA, Rome, Italy. In April 1996, he joined the faculty at the Department of Electronic Engineering,

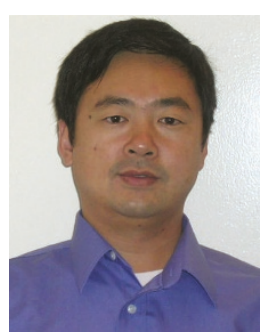
University of Electronic Science and Technology of China, where he has been an Associate Professor since July 1996. He was with the Department of Electrical and Computer Engineering, University of Florida, Gainesville, Fla, USA, as a Postdoctoral Research Associate from November 1998 to August 2000 and from September 2001 to March 2005. From August 2000 to August 2001, he was a Senior Research Associate at the Department of Electronic and Computer Engineering, Illinois Institute of Technology, Chicago, Ill, USA, as well as a Contractor at the Global Software Group, Motorola Inc., Elk Grove Village, Ill, USA. He has been a principal Systems Engineer with BAE Systems, Los Angeles, Calif, USA, since March 2005.
He is a Senior Member of IEEE. His expertise and research interest include LFM-CW radar system analysis and design, SAR/ISAR signal processing and image formation, polarimetric SAR, spectral analysis, array signal processing, and automatic target recognition.

Yi Jiang receive his B.S. degree in electrical engineering and information science from the University of Science and Technology of China (USTC), Hefei, China, in 2001. He received the M.S. and Ph.D. degrees in electrical engineering from the University of Florida, Gainesville, Fla, USA, in 2003 and 2005, respectively. In the summer of 2005, he worked as a Research Consultant in the Information System Technology Inc. (ISTI), Fort Collins, Colo, USA. He is now a Postdoctoral Researcher at the University of Colorado, Boulder, Colo, USA. His research interests are in the areas of signal processing, wireless communications, and information theory.

Hong Xiong received the B.S. degree in electrical engineering from Nanjing Universify of Aeronautics and Astronautics, Nanjing, China, in 1988, and the M.S. degree in electrical engineering from University of Electronic Science and Technology of China (UESTC), Chengdu, China, in 1991. She also received the M.S. degree in electrical engineering from University of Florida, Gainesville, Fla, in 2003.

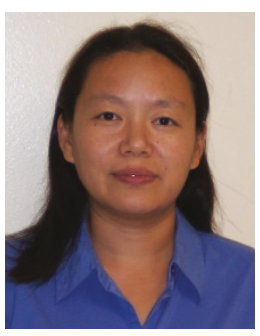
From April 1991 to August 1999, she was a Lecturer with the Department of Electrical Engineering at UESTC. She is now working towards the Ph.D degree in electrical engineering at the University of Florida. Her research interests include spectral analysis, adaptive and robust beamforming, and signal detection.

Jian Li received the M.S. and Ph.D. degrees in electrical engineering from The Ohio State University, Columbus, in 1987 and 1991, respectively. From July 1991 to June 1993, she was an Assistant Professor with the Department of Electrical Engineering, University of Kentucky, Lexington. Since August 1993, she has been with the Department of Electrical and Computer Engineering, University of Florida, Gainesville,

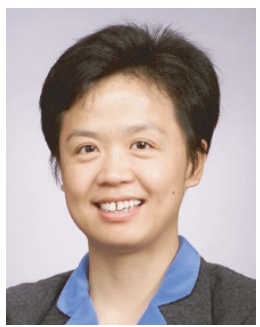
where she is currently a Professor. Her current research interests include spectral estimation, statistical and array signal processing, sensor networks, machine learning, and their applications. She is a Fellow of IEEE and a Fellow of IEE. She received the 1994 National Science Foundation Young Investigator Award and the 1996 Office of Naval Research Young Investigator Award. She was an Associate Editor of the IEEE Transactions on Signal Processing from 1999 to 2005. She has been an Associate Editor of the IEEE Signal Processing Magazine since 2003. She is presently a Member of two of the IEEE Signal Processing Society Technical Committees: the Signal Processing Theory and Methods (SPTM) Technical Committee and the Sensor Array and Multichannel (SAM) Technical Committee. 
Geoffrey A. Barrall received the B.S. degree in chemistry and mathematics from the University of California Davis in 1990 and the Ph.D. degree in physical chemistry from the University of California Berkeley in 1995. He is currently the Technology Leader for GE Security in San Diego. In this role, he is responsible for leading the development of quadrupole resonance and low-frequency magnetic sensing technolo-

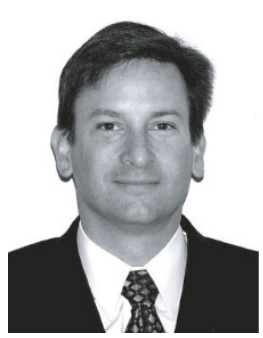
gies and products for GE Security. He has been exploring the use of quadrupole resonance for the detection of explosives in both landmine and security applications for the past 9 years. His contributions have spanned the theoretical, experimental, and signal processing aspects of QR detection. 